\title{
Author Index for Volume 66
}

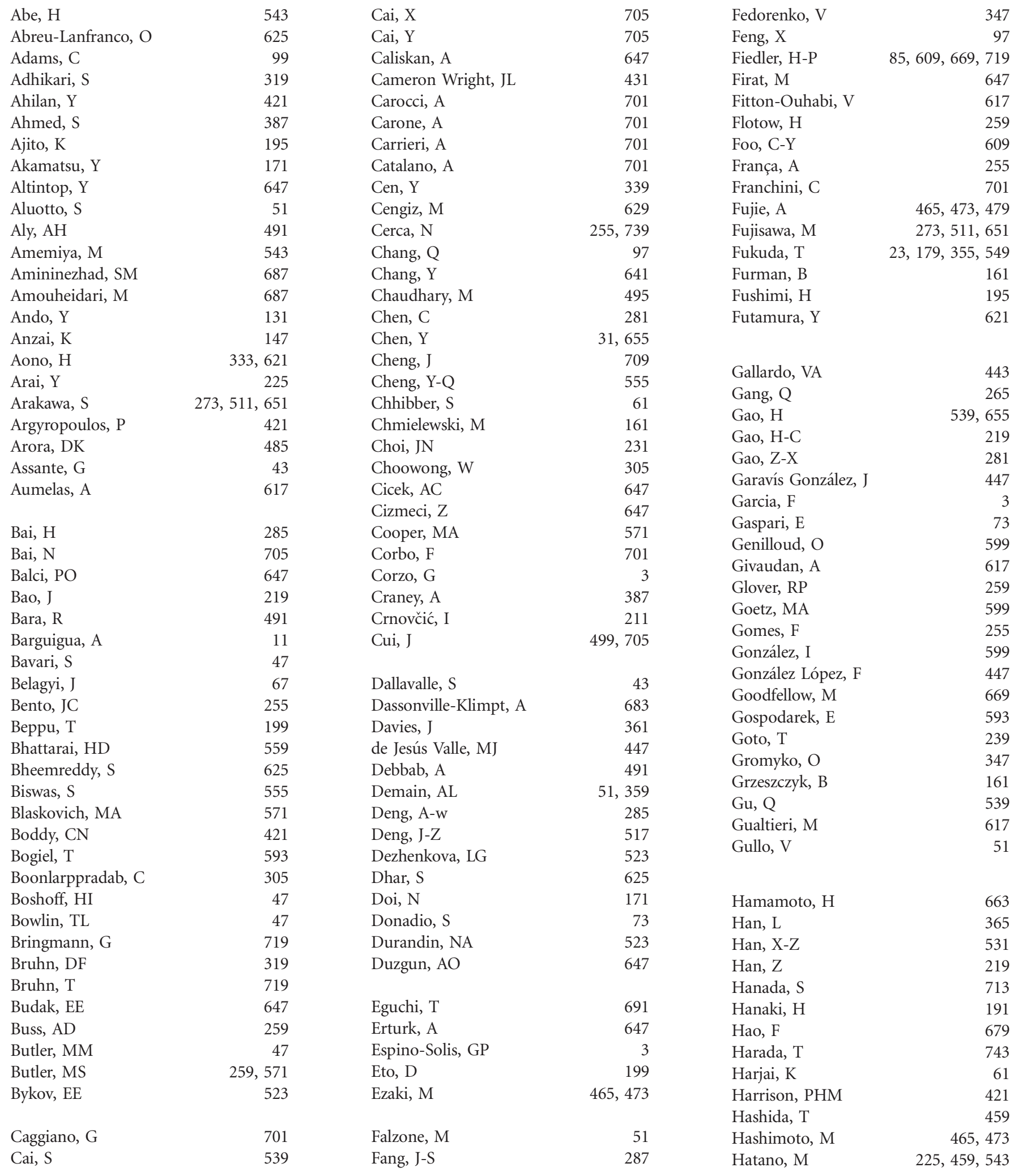


Hattori, S

Hayakawa, K

Hayakawa, Y

Hayashi, C

He, F

$\mathrm{He}, \mathrm{J}$

Helaly, SE

Hennessy, E

Herath, K

Hirai, Y

Hiraoka, Y

Hirose, $T$

Hongbin, Z

Hosoya, T

Houard, J

Hsu, A

Hsu, D-S

$\mathrm{Hu}, \mathrm{C}-\mathrm{Q}$

$\mathrm{Hu}, \mathrm{F}$

$\mathrm{Hu}, \mathrm{J}-\mathrm{H}$

$\mathrm{Hu}, \mathrm{L}-\mathrm{f}$

Huang, $\mathrm{H}$

Huang, K-J

Huang, Y

Hurdle, JG

Hwang, S

\section{Igarashi, M}

Igarashi, Y

Iinuma, $\mathrm{H}$

Ikeda, D

Ikeda, M

Ikeda, Y

Imachi, $M$

Imamura, $\mathrm{N}$

Imhoff, JF

Imoto, $\mathrm{M}$

Inahashi, Y

Inamura, $\mathrm{N}$

Indananda, C

Inokoshi, J

Inoue, $\mathrm{H}$

Inoue, $\mathrm{K}$

Ishikawa, K

Ishiyama, A

Itoh, M

Iwatsuki, M

Izawa, $\mathrm{M}$

Izumikawa, M

171, 225, 459, 543

531, 675

243, 543

675

277

85, 609, 669, 719

79, 311, 633

465, 473, 479

675

37, 549

171, 459

299, 563, 567, 727

191, 311

299, 563, 567, 727

Jamali, L

Jayasuriya, H

Jensen, SE

Jeon, B

Jetter, $P$

Ji, Z

Jia, A

Jiang, W

Jiang, Y-L

Jiang, Z-K

Jin, J
225

225

295

165

543

171

191

731

Jonet, A

Ju, J

Kai, $\mathrm{H}$

Kalyon, B

Kamiya, Y

Kanai, M

Kanasaki, R

Kaneko, K

Kawada, M

Kawaguchi, M

Kawahara, T

Kawakita, E

Kawasaki, Hiroko

Kawasaki, Hisashi

Kawatani, M

Kaye, KS

Keller, U

Kim, J

Kim, JG

Kim, JH

Kim, T

Kimura, T

Kino, $\mathrm{H}$

Kinoshita, N

Kirst, HA

Kitagawa, Masayuki

Kitagawa, Mitsuhiro

Kitamura, K

Knauer, M

Kobayashi, J

Kobayashi, K

Kobayashi, T

Kocsubé, S

Komagata, D

Komatsuki, Y

Koshino, $\mathrm{H}$

Koshiro, N

Kotturi, SR

Koyama, N

Koyama, Y

$\mathrm{Ku}, \mathrm{H}$

Kubota, T

Kudo, F

Kulik, A

Kumagai, H

Kumar, M

Kumar, S

Kumura, K

Kuzmin, VA

Kwiecińska-Piróg, J

599

401

351

719

265

679

339

531

281

281

Lane, D

Lear, MJ

Lee, $\mathrm{CH}$

Lee, $\mathrm{H}-\mathrm{S}$

Lee, JJ

Lee, JS

Lee, MA

Lee, RE

Lee, Y-J
$465,473,479$

609

311

663

473

663

543

$17,23,179$

299, 563, 567, 727

147

713

333

$247,333,621$

625

211

231

231

89

559

459

459

225, 459, 543

105

743

165

131

719

277

179

731

67

743

$$
171
$$

247, 621

333

259

$$
303
$$

171

453

277

691

85

243

319

495

195

523

593
Legendre, C

Leong, CY

Lephart, PR

Li, B

Li, D

Li, J-b

Li, J-S

Li, J-Y

Li, L

Li, S

Li, W-J

Li, X

Li, Y

Lian, Y

Liang, B

Lim, C

Lin, M-R

Lin, S-H

Liu, C

Liu, J-M

Liu, L-g

Liu, N

Liu, Q-F

Liu, S-W

Liu, $\mathrm{X}$

Liu, Z-P

Lu, X

Luzhetskyy, A

Maddox, MM

Madduri, $\mathrm{K}$

Maezawa, Y

Maffioli, SI

Magarvey, NA

Maggio, C

Magi, S

Manderscheid, N

Marchaim, D

Marona, $\mathrm{H}$

Martin, ET

Martín, J

Martino, PA

Maruyama, $\mathrm{H}$

Masaki, S

Masuda, T

Masuma, R

Masurekar, P

Matsui, $\mathrm{H}$

Matsumoto, A

Matsumoto, $\mathrm{T}$

Matsunaga, S

Miragaia, M

Miyachi, N

Miyake, T

Miyanaga, S

Moir, DT

Mojid Mondol, MA

Monciardini, P

Montagna, MT

Mooij, MJ

Morino, $\mathrm{T}$

99

259

625

339

539

709

735

281

205

31, 499

205

443

443

655

705

231

453

453

679

281

709

679

287

281

339, 499, 555 


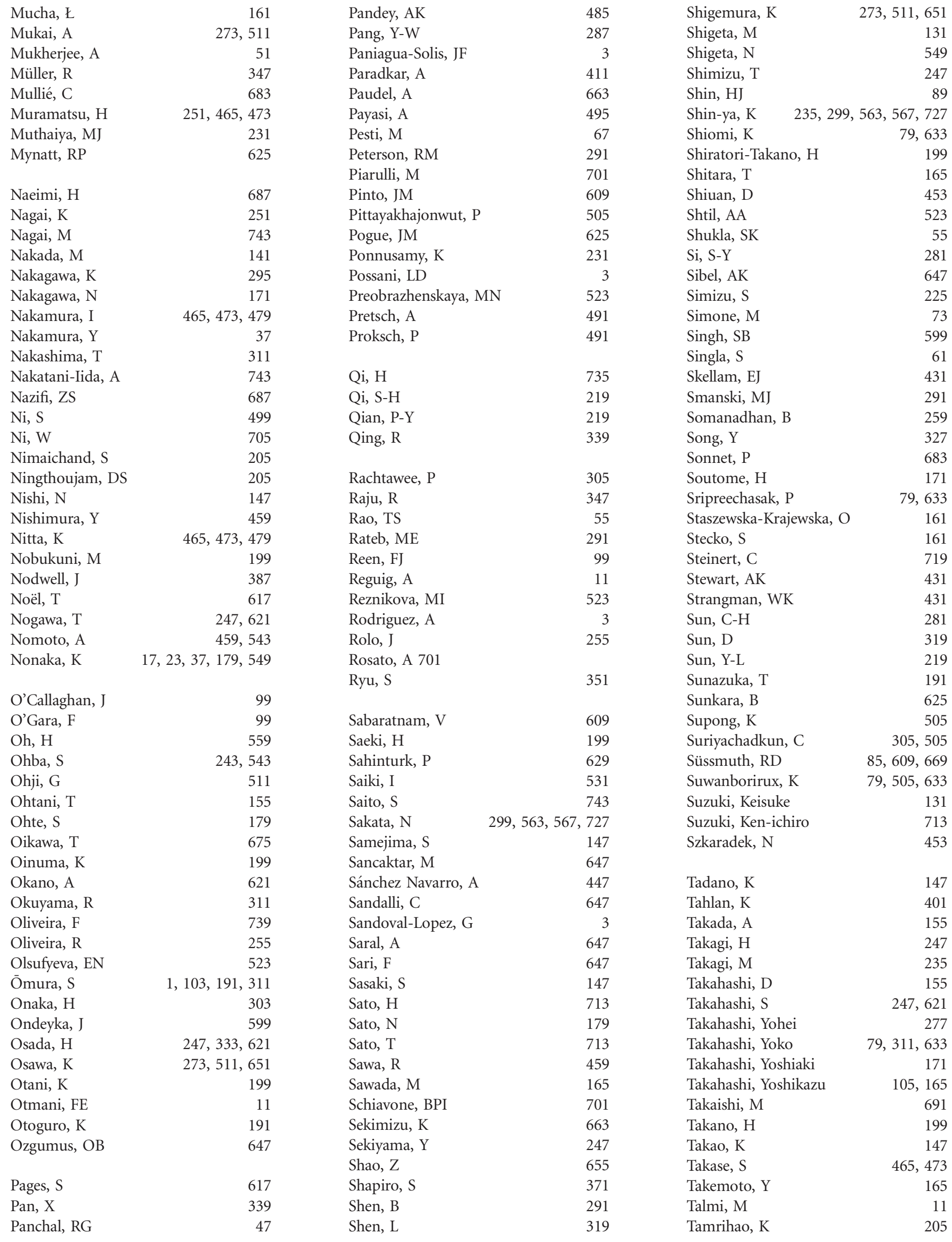




\begin{tabular}{|c|c|c|c|c|c|}
\hline Tamura, $\mathrm{T}$ & 713 & Vater, J & 211 & Yao, S-C & 641 \\
\hline Tanasupawat, S & 79,633 & Villegas, E & 3 & Yasuhara, T & 179 \\
\hline Tang, L-Q & 531 & Virág, E & 67 & Yildiz, N & 647 \\
\hline Tang, S-K & 205 & & & Yim, JH & 559 \\
\hline Tang, $\mathrm{X}$ & 655 & Wakiyama, Y & 195 & Ying, L-p & 285 \\
\hline Tatsuta, K & 107 & Wang, C & 655 & Yoshida, $\mathrm{T}$ & 195 \\
\hline Teixeira, $\mathrm{P}$ & 255 & Wang, F-F & 281 & Yoshikawa, K & $465,473,479$ \\
\hline Teramoto, $\mathrm{K}$ & 713 & Wang, J-D & $285,287,735$ & Yotsumoto, M & 303 \\
\hline Tevyashova, AN & 523 & Wang, N & 641 & You, X-F & 281 \\
\hline Thamchaipenet, A & 675 & Wang, R & 705 & $\mathrm{Yu}, \mathrm{L}$ & 97 \\
\hline & $303,355,549$ & Watanabe, Kenta & 199 & Zhang, C & 31,599 \\
\hline Tormo, JR & 599 & Watanabe, Kunitomo & 239 & Zhang, G & $31,539,719$ \\
\hline Toshima, $\mathrm{K}$ & 155 & Watanabe, $\mathrm{M}$ & $465,473,479$ & Zhang, $\mathrm{H}$ & $31,285,287$ \\
\hline Tran, CM & 239 & Watanabe, Takafumi & 459 & Zhang, L-J & 287 \\
\hline Tsuchida, T & $299,563,567,727$ & Watanabe, Takashi & 195 & Zhang, Q & 31 \\
\hline Tsukamoto, S & 155 & Watanabe, Takumi & 543 & Zhang, S & 31,327 \\
\hline Tsutsui, A & 191 & Watanabe, Y & 131 & Zhang, W & 31 \\
\hline \multirow[t]{2}{*}{ Tynan, $\mathrm{H}$} & 51 & Wei, S & 265 & Zhang, X-Y & 219 \\
\hline & & Wiese, J & $85,609,719$ & Zhang, Y & 327,679 \\
\hline Uchida, R & $23,37,179,549$ & Wray, V & 491 & Zhang, Y-G & 205 \\
\hline Uda, K & 155 & $\mathrm{Wu}, \mathrm{L}$ & 499 & Zhang, Y-j & 285 \\
\hline Uruga, T & 147 & & & Zhu, T & 539 \\
\hline Usami, I & 543 & Yadav, AK & 485 & Zhu, W-Y & 205 \\
\hline Utsumi, R & 459 & Yamaji, K & 311 & Zhu, Y & 31 \\
\hline \multirow[t]{2}{*}{ Uwamori, M } & 141 & Yamamoto, M & $195,465,473,479$ & Zhu, Y-l & 709 \\
\hline & & Yanagida, Y & 459 & Zinecker, H & 669 \\
\hline Vágvölgyi, C & 67 & Yang, L-L & 205 & Zink, DL & 599 \\
\hline Vanner, S & 443 & Yang, Z & 679 & & \\
\hline Vardhan, S & 485 & Yao, F & 281 & & \\
\hline
\end{tabular}

